\title{
Non-linear effects on radiation propagation around a charged compact object
}

\author{
R.R. Cuzinatto ${ }^{1}$ C.A.M. de Melo ${ }^{1,2}$ - K.C. de Vasconcelos ${ }^{3}$ L.G. Medeiros ${ }^{3}$. \\ P.J. Pompeia ${ }^{4,5}$
}

Received: 8 July 2015 / Accepted: 12 September 2015 / Published online: 23 September 2015

(c) Springer Science+Business Media Dordrecht 2015

\begin{abstract}
The propagation of non-linear electromagnetic waves is carefully analyzed on a curved spacetime created by static spherically symmetric mass and charge distribution. We compute how non-linear electrodynamics affects the geodesic deviation and the redshift of photons propagating near this massive charged object. In the first order approximation, the effects of electromagnetic self-interaction can be distinguished from the usual Reissner-Nordström terms. In the particular case of Euler-Heisenberg effective Lagrangian, we find that these self-interaction effects might be important near extremal compact charged objects.
\end{abstract}

Keywords Non-linear electrodynamics · Black holes · Redshift $\cdot$ Light bending $\cdot$ Geodesic path

$凶$ C.A.M. de Melo

cassius.anderson@gmail.com

1 Instituto de Ciência e Tecnologia, Universidade Federal de Alfenas, Rod. José Aurélio Vilela (BR 267), Km 533, No. 11999 , CEP 37701-970, Poços de Caldas, MG, Brazil

2 Instituto de Física Teórica, Universidade Estadual Paulista, Rua Bento Teobaldo Ferraz 271 Bloco II, P.O. Box 70532-2, CEP 01156-970, São Paulo, SP, Brazil

3 Departamento de Física Teórica e Experimental and Escola de Ciência e Tecnologia, Universidade Federal do Rio Grande do Norte, Campus Universitário, s/n—Lagoa Nova, CEP 59078-970, Natal, RN, Brazil

4 Instituto de Fomento e Coordenação Industrial, Departamento de Ciência e Tecnologia Aeroespacial, Praça Mal, Eduardo Gomes 50, CEP 12228-901, São José dos Campos, SP, Brazil

5 Instituto Tecnológico de Aeronaútica, Departamento de Ciência e Tecnologia Aeroespacial, Praça Mal, Eduardo Gomes 50, CEP 12228-900, São José dos Campos, SP, Brazil

\section{Introduction}

Generalizations of Maxwell electrodynamics have been proposed since it was established and they are motivated by several reasons such as experimental constraints on the eventual photon mass (Tu et al. 2005; Cuzinatto et al. 2011; Bonin et al. 2010), classical aspects of vacuum polarization (Heisenberg and Euler 1936; Schwinger 1951), electrodynamics in the context of strings and superstrings (Seiberg and Witten 1999; Fradkin and Tseytlin 1985; Bergshoeff et al. 1987; Metsaev et al. 1987; Leigh 1989), etc. Among the several generalizations, there is a group known as Nonlinear Electrodynamics (NLED) which is characterized by presenting nonlinear field equations. Examples of NLED are Born-Infeld theory (Born 1934, 1937; Born and Infeld 1934; Stehle and DeBaryshe 1966) and Euler-Heisenberg electrodynamics (Heisenberg and Euler 1936). The former was proposed to limit the maximum value of the electric field of a point charge (Delphenich 2003) and the last arises as an effective action of one-loop QED (Dunne 2005).

Since the decade of 1980, several applications of NLED in the context of gravitation were proposed (Salazar et al. 1984; Demianski 1986; De Oliveira 1994; Novello et al. 2003; Barcelo et al. 2005; Cai et al. 2008), including applications to cosmology (Garcia-Salcedo and Breton 2000; De Lorenci et al. 2002; Dyadichev et al. 2002; Moniz 2002; Novello et al. 2004, 2009; Medeiros 2012) and spherically symmetric solutions of charged Black Holes $(\mathrm{BH})$ (Ayon-Beato and Garcia 1998; Yajima and Tamaki 2001; Bronnikov 2001; Diaz-Alonso and Rubiera-Garcia 2010a, 2010b, 2013a; Ruffini et al. 2013). Moreover, generalizations of Reissner-Nordström solution with NLED were studied where stability and thermodynamics properties of the BH were analyzed (Chemissany et al. 2008; Gunasekaran et al. 2012; Hendi and Vahidinia 2013; DiazAlonso and Rubiera-Garcia 2013b; Mo and Liu 2014; 
Bretón and Bergliaffa 2014; Hendi et al. 2014; Hendi and Momennia 2015; Hendi and Panahiyan 2014; Rasheed 1997). In particular, the geodesic motion of test particles around Born-Infeld BH was studied in Linares et al. (2014) and references therein. However, in most of the papers found in the literature the direct influence of the background electric field on radiation propagation is ignored.

In the early 1970's it was realized that the self-interaction of NLED modifies the dispersion relation of a photon propagating in a region with a background electric field (Bialynicka Birula and Bialynicki Birula 1970). From the geometrical point of view, this modification can be mapped to an effective metric in the flat spacetime (Boillat 1970; Novello et al. 2000; Novello and Goulart 2010; Lorenci and Souza 2001; Novello and Bittencourt 2012). This effective metric can be promptly generalized to a curved spacetime metric through the minimal coupling prescription. As a consequence, there are two simultaneous effects on radiation propagation: first, the path of light rays may be altered due to self-interaction of the electric field via the effective metric; second, the photon dynamics is affected by the spacetime curvature. In fact, some preliminary results concerning photon propagation in non-linear interaction with a background electric field and in the presence of a BH were obtained in the context of Euler-Heisenberg and Born-Infeld electrodynamics (De Lorenci et al. 2001; Bretón 2002). In the present work, the authors intend to generalize these results for a generic NLED and particularly show that, despite the fact that the background electric field does not generate effective horizons (horizons that would be sensed only by radiation), this field directly influences the geometric redshift and geodesic deviation.

The paper is organized as follows. In Sect. 2, the spherically symmetric solution for a generic NLED is determined. In Sect. 3, minimal coupling prescription is used to generalize the effective metric in flat spacetime to a curved one. In this section, it is also shown that the effective metric does not produce any new horizon. In Sect. 4, the influence of the background electric field in geometric redshift and geodesic deviation is analyzed. Final remarks are presented in Sect. 5.

\section{General solution for NLED}

The matter Lagrangian for a general NLED invariant under parity is:

$\mathcal{L}=\mathcal{L}\left(F, G^{2}\right)$,

where $F \equiv-\frac{1}{4} F^{\mu \nu} F_{\mu \nu}$ and $G \equiv-\frac{1}{4} \tilde{F}^{\mu \nu} F_{\mu \nu}$. The quantity $F_{\mu \nu}$ is the electromagnetic field tensor and $\widetilde{F}^{\mu \nu}$ is its dual: $\widetilde{F}^{\mu \nu} \equiv \frac{1}{2} \eta^{\mu \nu \rho \sigma} F_{\rho \sigma}$, where $\eta^{\mu \nu \rho \sigma}=\frac{\varepsilon^{\mu \nu \rho \sigma}}{\sqrt{-g}}$ is the totally anti- symmetric tensor constructed from the Levi-Civita symbol $\varepsilon^{\mu \nu \rho \sigma}$ and the determinant $g$ of the metric tensor $g_{\mu \nu} \cdot 1$

The energy-momentum tensor is given by:

$T_{\mu \nu}=\mathcal{L}_{F} F_{\mu \lambda} F_{\nu}^{\lambda}+G \mathcal{L}_{G} g_{\mu \nu}-\mathcal{L} g_{\mu \nu}$,

with $\mathcal{L}_{F} \equiv \frac{\partial \mathcal{L}}{\partial F}$ and $\mathcal{L}_{G} \equiv \frac{\partial \mathcal{L}}{\partial G}$.

The static spherically symmetric line element is:

$d s^{2}=e^{v(r)} d t^{2}-e^{\lambda(r)} d r^{2}-r^{2} d \theta^{2}-r^{2} \sin ^{2} \theta d \phi^{2}$,

where $v(r)$ and $\lambda(r)$ are functions determined by the gravitational field equations. In order to preserve spacetime symmetry, one supposes the matter content to be constituted by a static and spherically symmetric electric charge. Hence, only the components $F_{01}=-F_{10}=-E(r)$ of the field strength $F_{\mu \nu}$ are non-null. Consequently $G=0$. In this case, the asymptotically flat exterior Schwarzchild-de SitterNLED solution of Einstein equations is:

$v \nu(r)=-\lambda(r)=\ln \left(1-\frac{2 m}{r}+\frac{1}{r} S(r)-\frac{\Lambda}{3} r^{2}\right)$.

We consider $c=G_{N}=1$. The constant $m$ is the geometric mass, $\Lambda$ is the cosmological constant and

$S(r) \equiv 2 \int r^{2}\left(-\mathcal{L}_{F} E^{2}+\mathcal{L}\right) d r$.

The field equations for $F_{\mu \nu}$ are:

$\partial_{\nu}\left(\mathcal{L}_{F} \sqrt{-g} F^{\mu \nu}\right)=0 \quad$ and $\quad \partial_{[\rho} F_{\mu \nu]}=0$.

This implies:

$\mathcal{L}_{F} E=\frac{q}{r^{2}}$,

where $q$ is the total electric charge.

Note that an explicit solution can be obtained when an specific $\mathcal{L}\left(F, G^{2}\right)$ is chosen. For instance, Maxwell Electrodynamics is recovered when $\mathcal{L}_{F}=1$ and

$E=\frac{q}{r^{2}} \Rightarrow S(r)=\frac{q^{2}}{r}$

which is the well known Reissner-Nordström solution.

\section{Effective metric}

In NLED, the electromagnetic field is self-interacting and this is directly reflected on photon propagation. In particular, ${ }^{1}$ In flat spacetime, $F=\left(\vec{E}^{2}-\vec{B}^{2}\right) / 2$ and $G=\vec{E} \cdot \vec{B}$. The vectors $\vec{E}$
and $\vec{B}$ are the electric and magnetic fields respectively. F and $\mathrm{G}$ are the
only Lorentz and gauge invariant functions of $F_{\mu \nu}$ (Delphenich 2006). 
when radiation propagates in a slowly varying electromagnetic field as a background, the self-interaction between the radiation and the background field can be described by an effective metric: $\bar{\eta}^{\mu \nu}$ (Bialynicka Birula and Bialynicki Birula 1970; Boillat 1970; Novello et al. 2000). In flat spacetime one has

$\bar{\eta}^{\mu \nu} k_{\mu} k_{v}=\left(\eta^{\mu \nu}+\lambda_{ \pm} F^{\mu \beta} F_{\beta}^{v}\right) k_{\mu} k_{v}=0$,

where

$\lambda_{ \pm}=\frac{-\mathcal{L}_{F}\left(\mathcal{L}_{G G}+\mathcal{L}_{F F}\right)+2 F \overline{\mathcal{L}} \pm \sqrt{\delta}}{2\left[G^{2} \overline{\mathcal{L}}+2 \mathcal{L}_{F}\left(\mathcal{L}_{G G} F-\mathcal{L}_{F G} G\right)-\mathcal{L}_{F}^{2}\right]}$,

with

$$
\begin{aligned}
\delta=[ & {\left[2 F\left(\mathcal{L}_{F F} \mathcal{L}_{G G}-\mathcal{L}_{F G}^{2}\right)+\mathcal{L}_{F}\left(\mathcal{L}_{G G}-\mathcal{L}_{F F}\right)\right]^{2} } \\
& +\left[2 G\left(\mathcal{L}_{F F} \mathcal{L}_{G G}-\mathcal{L}_{F G}^{2}\right)-2 \mathcal{L}_{F} \mathcal{L}_{F G}\right]^{2}
\end{aligned}
$$

and

$\overline{\mathcal{L}}=\mathcal{L}_{F F} \mathcal{L}_{G G}-\mathcal{L}_{F G}^{2}$.

The signal \pm in Eq. (10) indicates birefringence (NiauAkmansoy and Medeiros 2014; Bialynicka Birula and Bialynicki Birula 1970), i.e. the possible dependence of the photon propagation on its polarization, which is a typical phenomenon of NLED. When minimal coupling prescription $\left(\eta_{\mu \nu} \rightarrow g_{\mu \nu}\right)$ is performed, the effective metric on the curved spacetime is found to be:

$\bar{g}^{\mu \nu}=g^{\mu \nu}+\lambda_{ \pm} F^{\mu \beta} F_{\beta}^{\nu}$,

where $g^{\mu v}$ is the spacetime metric given in Eq. (3)—see Appendix A of Novello et al. (2000) for more details. This way, photons (i.e. weak radiation propagating fields that do not disturb the spacetime) will follow geodesics described by the following effective line element

$d \bar{s}^{2}=\frac{e^{\nu}}{\left[1+\lambda_{ \pm} E^{2}\right]} d t^{2}-\frac{e^{-\nu}}{\left[1+\lambda_{ \pm} E^{2}\right]} d r^{2}-r^{2} d \Omega^{2}$.

This line element contains both spacetime (gravitational) effects-via the factor $e^{v}$-and the influence of the background field-through the function $\left[1+\lambda_{ \pm} E^{2}\right]$. The angular part of the line element is $d \Omega^{2}=d \theta^{2}+\sin ^{2} \theta d \phi^{2}$, as usual. It is also worth mentioning that the NLED under consideration influences photon trajectory both gravitationallythrough $S(r)$ —and electromagnetically—by means of $\lambda_{ \pm}$. For instance, in Maxwell electrodynamics: $\lambda_{ \pm}=0$ and $S(r)$ is given by Eq. (8). On the other hand, in Born-Infeld theory, where

$$
\mathcal{L}_{\mathrm{BI}}=b^{2}\left[1-\sqrt{1-\frac{2 F}{b^{2}}-\frac{G^{2}}{b^{4}}}\right],
$$

one finds $\lambda_{ \pm}=\frac{1}{b^{2}-E^{2}} ; S(r)$ is given by elliptic functions (Bretón 2002). Note that, both in Maxwell and Born-Infeld cases, no birefringence is present (Boillat 1970; De Melo et al. 2015).

The first point to be analyzed here concerns the existence of effective event horizons. For this goal, an investigation of the radial null geodesics is carried on using Eq. (14). The result is:

$$
\left(\frac{d t}{d r}\right)^{2}=\frac{\left[1+\lambda_{ \pm} E^{2}\right] e^{-v}}{\left[1+\lambda_{ \pm} E^{2}\right] e^{v}}=e^{-2 v},
$$

which is exactly the same expression obtained considering propagation with the spacetime metric Eq. (3). This means that the horizons "seen" by photons are generated exclusively by gravitational effects of the spacetime. Note that this result is formally independent of the particular type of NLED under consideration because the factors scaling with $\lambda_{ \pm}$cancel out in Eq. (16). However, we emphasize that the gravitational effects of the NLED are still present in $v$.

Although the horizons do not depend on the effective metric, the later is important in other phenomena such as geometric redshift and light deflection. These two effects are analyzed in the following section.

\section{Influence of NLED on radiation propagation}

In this section, an investigation on how an specific NLED influences the radiation redshift and geodesic deviation will be conducted. No influence of the cosmological constant will be considered here, i.e. $\Lambda=0$. A perturbative approach will be adopted, in which nonlinear effects are small corrections to the Maxwell theory. $\mathcal{L}\left(F, G^{2}\right)$ is written as

$\mathcal{L}\left(F, G^{2}\right) \simeq F+\frac{1}{2} a_{+} F^{2}+\frac{1}{2} a_{-} G^{2}+\mathcal{O}(3)$

where $\mathcal{O}(3)$ represents higher order terms and

$a_{+} F \sim a_{-} G \ll 1$.

The condition $a_{ \pm}>0$ ensures that the energy density is positive definite (Diaz-Alonso and Rubiera-Garcia 2010a).

In the first order regime of perturbation and considering a radial electric field ( $F=\frac{E^{2}}{2}$ e $G=0$ ), Eq. (10) reduces to:

$$
\begin{aligned}
& \lambda_{+} \simeq \frac{\mathcal{L}_{F F}}{\mathcal{L}_{F}} \simeq a_{+}\left(1-\frac{a_{+}}{2} E^{2}\right), \\
& \lambda_{-} \simeq \frac{\mathcal{L}_{G G}}{\mathcal{L}_{F}-2 F \mathcal{L}_{G G}} \simeq a_{-}\left(1-\frac{a_{+}}{2} E^{2}+a_{-} E^{2}\right) .
\end{aligned}
$$

Hence,

$$
1+\lambda_{ \pm} E^{2} \simeq 1+a_{ \pm} E^{2} .
$$


The next step is to obtain $S(r)$. By substituting Eq. (17) into Eq. (7), one obtains:

$$
\left(1+\frac{a_{+}}{2} E^{2}\right) E \simeq \frac{q}{r^{2}}
$$

which leads to:

$E \simeq \frac{q}{r^{2}}\left[1-\frac{a_{+}}{2}\left(\frac{q}{r^{2}}\right)^{2}\right]$

Notice that the condition (18) implies:

$a_{+}\left(\frac{q}{r^{2}}\right)^{2} \ll 1$

Now Eq. (22) is substituted in Eq. (5) leading to

$S(r) \simeq \frac{q^{2}}{r}-\frac{k}{20} \frac{q^{4}}{r^{5}}$

where $k \equiv a_{+}$. The change of notation $k$ instead of $a_{+}$ is done in order to distinguish gravitational effects of the NLED from purely electromagnetic effects, i.e. $k$ is related to the spacetime metric and $a_{ \pm}$to the effective metric.

Finally, the components of the metric Eq. (3) in the first order approximation are rewritten as:

$$
\begin{aligned}
& \bar{g}_{00}=\frac{e^{v}}{\left[1+\lambda_{ \pm} E^{2}\right]} \simeq W(r)-\frac{k}{20} \frac{q^{4}}{r^{6}}-a_{ \pm}\left(\frac{q}{r^{2}}\right)^{2}, \\
& \frac{1}{\bar{g}_{11}}=\frac{\left[1+\lambda_{ \pm} E^{2}\right]}{-e^{-v}} \simeq-\left(W(r)-\frac{k}{20} \frac{q^{4}}{r^{6}}+a_{ \pm}\left(\frac{q}{r^{2}}\right)^{2}\right),
\end{aligned}
$$

where

$W(r)=1-\frac{2 m}{r}+\frac{q^{2}}{r^{2}}$

is the usual Reissner-Nordström term.

In this approximation, the manner by which the event horizon is affected by the NLED can be studied. The horizons $r_{ \pm}$are obtained when the condition $g_{11}=0$ is considered, which in our particular case gives:

$1-\frac{2 m}{r}+\frac{q^{2}}{r^{2}}-\frac{k}{20} \frac{q^{4}}{r^{6}} \simeq 0$.

The approximated solution to this equation is:

$r_{ \pm} \simeq r_{ \pm(0)} \pm \frac{r_{ \pm(0)}^{2}}{40 \sqrt{m^{2}-q^{2}}} k \frac{q^{4}}{r_{ \pm(0)}^{6}}$

where

$r_{ \pm(0)}=m \pm \sqrt{m^{2}-q^{2}}$ is the usual Reissner-Nordström horizon. Notice that

$r_{+}>r_{+(0)}$ and $r_{-}<r_{-(0)}$.

Hence, the effect of the nonlinearity of NLED is to displace the external horizon outwards and the internal horizon inwards.

\subsection{Geometric redshift}

The geometric redshift of a light ray emitted radially at $r_{1}$ and detected at $r_{2}$ with $r_{+}<r_{1}<r_{2}$, is given by

$1+z=\sqrt{\frac{\bar{g}_{00}\left(r_{2}\right)}{\bar{g}_{00}\left(r_{1}\right)}}$.

By using Eq. (25) and considering $r_{1}=r$ and $r_{2} \rightarrow \infty$, one gets:

$1+z \simeq\left[1-\frac{2 m}{r}+\frac{q^{2}}{r^{2}}-\frac{k}{20} \frac{q^{4}}{r^{6}}-a_{ \pm}\left(\frac{q}{r^{2}}\right)^{2}\right]^{-1 / 2}$

The first three terms of the right hand side of this equation correspond to the usual Reissner-Nordström terms where the redshift is increased by the mass while the charge (Maxwell term) is responsible for decreasing it. The last two terms describe the effects of the NLED: the fourth one is a consequence of the curvature of spacetime generated by non-linear electromagnetic terms and the fifth one is due to the effective metric of the NLED, i.e., a redshift created by photons interacting with the background electric field. Note that both terms lead to an increasing of $z$. It is also worth mentioning that the influence of the effective metric makes redshift dependent on the polarization since in general $a_{+} \neq a_{-}$.

If a weak field approximation is taken into account, i.e.

$\frac{2 m}{r} \ll 1 \quad$ and $\quad \frac{q^{2}}{r^{2}} \lll 1$,

then Eq. (33) is simplified to

$z \simeq \frac{m}{r}+\frac{3}{2} \frac{m^{2}}{r^{2}}-\frac{q^{2}}{2 r^{2}}+\frac{a_{ \pm}}{2}\left(\frac{q}{r^{2}}\right)^{2}$,

where the mass dependence is taken up to second order and the term $k\left(\frac{q}{r^{2}}\right)^{2} \frac{q^{2}}{r^{2}}$ is neglected, meaning that the curvatureborn term disappears.

The magnitude of the last term in Eq. (35) depends on the coupling constant of the NLED, which is a free parameter (up to constraints coming from experimental data). For instance, for Born-Infeld Lagrangian, Eq. (15),

$a_{+}=a_{-}=\frac{1}{b^{2}}$ 
The exception is Euler-Heisenberg electrodynamics which is obtained as an effective theory at the low-energy regime of QED. ${ }^{2}$ According to Bialynicka Birula and Bialynicki Birula (1970), Euler-Heisenberg Lagrangian is given by

$\mathcal{L}_{\mathrm{EH}} \simeq F+\frac{2 \alpha^{2} \hbar^{3}}{45 m_{e}^{4}}\left(4 F^{2}+7 G^{2}\right)$,

where $\alpha$ is the fine-structure constant and $m_{e}$ is the electron mass. This way,

$a_{+}=\frac{16 \alpha^{2} \hbar^{3}}{45 m_{e}^{4}}, \quad a_{-}=\frac{28 \alpha^{2} \hbar^{3}}{45 m_{e}^{4}}$,

where $a_{+} \sim a_{-} \sim 10^{-28} \frac{\mathrm{cm}^{3}}{\mathrm{erg}}$.

In order to have an idea of the magnitude of the nonlinear term in the context of Euler-Heisenberg electrodynamics, a hypothetical situation is considered: a star like the Sun (with the same radius $R_{\odot}$ and mass $M_{\odot}$ ) with an electric charge producing an extremal black hole, i.e. $m=q$. This is the maximal charge permitted for a static black hole if the cosmic censorship hypothesis is considered. In this case,

$$
\begin{aligned}
& \frac{m}{R_{\odot}} \rightarrow \frac{M_{\odot} G}{c^{2} R_{\odot}} \simeq 2 \times 10^{-6} \Rightarrow \frac{m^{2}}{R_{\odot}^{2}} \sim \frac{q^{2}}{R_{\odot}^{2}} \sim 10^{-12}, \\
& \frac{a_{ \pm}}{2}\left(\frac{q}{R_{\odot}^{2}}\right)^{2} \rightarrow \frac{a_{ \pm}}{2} G\left(\frac{M_{\odot}}{R_{\odot}^{2}}\right)^{2} \simeq 4 \times 10^{-15} .
\end{aligned}
$$

For this hypothetical situation, the nonlinear term (scaling with $a_{ \pm}$) is nine orders of magnitude smaller than the dominant term $\frac{m}{r}$. Although being negligible this term becomes rapidly important as $r$ decreases. In particular, for $R_{\mathrm{WD}}=10^{-3} R_{\odot}$ (a white dwarf radius) the nonlinear term becomes of the same magnitude order of $\frac{m}{r}$, i.e.

$$
\begin{aligned}
& \frac{m}{R_{\mathrm{WD}}} \sim \frac{a_{ \pm}}{2}\left(\frac{q}{R_{\mathrm{WD}}^{2}}\right)^{2} \sim 10^{-3} \\
& \frac{m^{2}}{R_{\mathrm{WD}}^{2}} \sim \frac{q^{2}}{R_{\mathrm{WD}}^{2}} \sim 10^{-6} .
\end{aligned}
$$

This might indicate an astrophysical object for which the NLED effects could be important indeed.

Next, the geodesic deviation will be analyzed.

\subsection{Geodesic deviation of radiation}

The geodesic deviation of a light ray propagating from infinity to a point at a distance $r$ from the origin of our coordinate

\footnotetext{
${ }^{2}$ Energies much less than that of the electron rest energy.
}

system in a region with static spherically symmetric gravitational and electric fields is given by (Weinberg 1972):

$\phi(r)-\phi_{\infty}=\int_{r}^{\infty} \sqrt{\frac{\bar{g}_{11}(r)}{\left[\left(\frac{r}{r_{0}}\right)^{2}\left(\frac{\bar{g}_{00}\left(r_{0}\right)}{\bar{g}_{00}(r)}\right)-1\right]}} \frac{d r}{r}$,

where $r_{0}$ is the maximum approximation distance of the light ray from the gravitational/electric source. In the approximation of small corrections to Maxwell theory $\bar{g}_{00}$ and $\bar{g}_{11}$ are given by Eqs. (25) and (26), respectively. In first order, the above expression is rewritten as

$$
\begin{aligned}
& \left(\phi(r)-\phi_{\infty}\right)_{ \pm} \\
& \simeq \int_{r}^{\infty} f_{1}(r)\left\{1-a_{ \pm}\left(\frac{q}{r^{2}}\right)^{2} f_{2}(r)+k \frac{q^{2}}{r^{2}}\left(\frac{q}{r^{2}}\right)^{2} f_{3}(r)\right\} d r
\end{aligned}
$$

where

$$
\begin{aligned}
& f_{1}(r)=\frac{1}{r}\left[\left(\frac{r}{r_{0}}\right)^{2} W\left(r_{0}\right)-W(r)\right]^{-1 / 2}, \\
& f_{2}(r)=1+\frac{1}{2} \frac{W\left(r_{0}\right)-\left(\frac{r}{r_{0}}\right)^{4} W\left(r_{0}\right)}{W\left(r_{0}\right)-\left(\frac{r_{0}}{r}\right)^{2} W(r)}, \\
& f_{3}(r)=\frac{1}{20 W(r)}\left[1-\frac{1}{2} \frac{W\left(r_{0}\right)-\left(\frac{r}{r_{0}}\right)^{6} W(r)}{W\left(r_{0}\right)-\left(\frac{r_{0}}{r}\right)^{2} W(r)}\right] .
\end{aligned}
$$

In Eq. (44) the first term is the standard effect of classical electrostatics in the context of general relativity, the second term is the contribution from NLED effective metric, and the third one is the correction due to spacetime curvature coming from the nonlinear background electric field. Note that the presence of the second term indicates that the geodesic deviation is dependent on the polarization of the radiation.

The integral in Eq. (44) can be evaluated analytically if the weak field-approximation (34) is considered:

$$
\begin{aligned}
\left(\phi(r)-\phi_{\infty}\right)_{ \pm} \simeq & \arcsin \left(\frac{r_{0}}{r}\right)+\frac{m}{r_{0}} h_{1}(r) \\
& -\frac{q^{2}}{2 r_{0}^{2}} h_{2}(r)+\frac{1}{2} a_{ \pm}\left(\frac{q}{r_{0}^{2}}\right)^{2} h_{3}(r)
\end{aligned}
$$

where

$$
\begin{aligned}
h_{1}(r)=2 & -\sqrt{1-\left(\frac{r_{0}}{r}\right)^{2}}-\sqrt{\frac{1-\frac{r_{0}}{r}}{1+\frac{r_{0}}{r}}} \\
h_{2}(r)= & 1-\frac{1}{2}\left(\frac{r_{0}}{r}\right) \sqrt{1-\left(\frac{r_{0}}{r}\right)^{2}} \\
& +\frac{1}{2} \arcsin \left(\frac{r_{0}}{r}\right)-\sqrt{\frac{1-\frac{r_{0}}{r}}{1+\frac{r_{0}}{r}}}
\end{aligned}
$$




$$
\begin{aligned}
h_{3}(r)= & \frac{9}{8} \arcsin \left(\frac{r_{0}}{r}\right)-\frac{1}{8}\left(\frac{r_{0}}{r}\right) \sqrt{1-\left(\frac{r_{0}}{r}\right)^{2}} \\
& +\frac{1}{4} \sqrt{1-\left(\frac{r_{0}}{r}\right)^{2}}\left(\frac{r_{0}}{r}\right)^{3} .
\end{aligned}
$$

The deviation observed in a region far from the source is given by:

$\Delta \phi=2\left|\phi\left(r_{0}\right)-\phi_{\infty}\right|-\pi$,

resulting:

$\Delta \phi_{ \pm} \simeq 4 \frac{m}{r_{0}}-\frac{q^{2}}{r_{0}^{2}}\left[1+\frac{\pi}{4}\right]+a_{ \pm} \frac{9 \pi}{16}\left(\frac{q}{r_{0}^{2}}\right)^{2}$.

This equation shows clearly the contributions of mass, electric charge and the nonlinear term for the evaluation of geodesic deviation in the weak-field approximation. In the case of the Euler-Heisenberg Lagrangian, Eq. (37), the contribution of each term can be evaluated by considerations analogous to those of the previous section. Preliminary results of the influence of the vacuum polarization effects described by Euler-Heisenberg electrodynamics were obtained in De Lorenci et al. (2001).

Particular cases are obtained if one takes one or more terms of Eq. (53) to be null. For instance, for an object of null charge the known result $\Delta \phi_{ \pm}=\frac{4 m}{r_{0}}$ is obtained. An interesting case is obtained when only the effect due to NLED self-interaction (the interaction of the radiation with the background electric field) is considered, i.e. no gravitational effects are taken into account. Essentially, the metric considered in this calculation is the effective metric in a flat spacetime. Mathematically, this corresponds to take $W(r)=1$ and $k=0$ in Eq. (44). The integration of this expression leads to a purely electric (PE) geodesic deviation:

$\Delta \phi_{ \pm}^{\mathrm{PE}} \simeq a_{ \pm} \frac{9 \pi}{16}\left(\frac{q}{r_{0}^{2}}\right)^{2}$

Hypothetically, this result can be used to verify experimentally if vacuum polarization is able to produce light deviation, as predicted by Eq. (54).

\section{Conclusion}

In this paper we have constructed the solution of Einstein equations for the exterior of spherically symmetric mass and charge distributions in the context of non-linear electrodynamics. The resulting line element is given in Eqs. (3), (4) and (5). It is asymptotically de Sitter and depends on the mass $m$, on the cosmological constant $\Lambda$, and on the functional $S(r)$. This object is an integral given in terms of the electrostatic field $\vec{E}$ and the Lagrangian density $\mathcal{L}\left(F, G^{2}\right)$ of the particular NLED to be taken into account.

First, we noticed that the radial null geodesics followed by photons in the curved effective metric can be calculated as usual, being the NLED influence encapsulated in function $v(r)$ via $S(r)$. The non-linearity of electrodynamics appears explicitly in Eq. (22) for the magnitude of the electric field, which is calculated when small perturbations of Maxwell theory are considered. This leads to a modification in the horizons of the charged $\mathrm{BH}$ as perceived by the photons: displacing the internal and external horizons inwards and outwards respectively.

The redshift of spectral lines is increased by the presence of the non-linear electrodynamic terms. Moreover, $z$ is sensible to the polarization of the radiation due to selfinteraction. In order to estimate the magnitude of the effects on $z$ coming from NLED, we decide to choose a particular non-linear theory and study it in the weak-field regime. Using Euler-Heisenberg Lagrangian, one concludes that extremal compact charged objects could produce redshifts for which the contribution from the ordinary $(m / r)$ term is as important as the NLED-born term.

Finally, we calculated the geodesic deviation experienced by radiation nearby massive charged bodies. The ReissnerNordström result is recovered along with small corrections to Maxwell electrodynamics due to NLED. The background electric field affects the geodesic path of light rays, increasing the deviation that would be expected from the linear theory. We also obtained an expression for the deviation when only the effect due to NLED self-interaction is taken into account-see Eq. (54). This could be used for comparison with observational data in order to determine if the vacuum polarization actually can bend light rays.

Acknowledgements KCV acknowledges CAPES-Brazil for financial support. LGM is grateful to FAPERN-Brazil for financial support.

\section{References}

Ayon-Beato, E., Garcia, A.: Phys. Rev. Lett. 80(23), 5056 (1998)

Barcelo, C., Liberati, S., Visser, M.: Living Rev. Relativ. 8, 12 (2005)

Bergshoeff, E., Sezgin, E., Pope, C.N., Townsend, P.K.: Phys. Lett. B 188(1), 70 (1987)

Bialynicka Birula, Z., Bialynicki Birula, I.: Phys. Rev. D 2(10), 2341 (1970)

Boillat, G.: J. Math. Phys. 11(3), 941 (1970)

Bonin, C.A., Bufalo, R., Pimentel, B.M., Zambrano, G.E.R.: Phys. Rev. D 81(2), 025003 (2010)

Born, M.: Proc. R. Soc. Lond. Ser. A 143, 410 (1934)

Born, M.: Ann. Inst. Henri Poincaré 7, 155 (1937)

Born, M., Infeld, L.: Proc. R. Soc. Lond. Ser. A 144(852), 425 (1934)

Bretón, N.: Class. Quantum Gravity 19(4), 601 (2002)

Bretón, N., Bergliaffa, S.E.P.: (2014, preprint)

Bronnikov, K.A.: Phys. Rev. D 63(4), 044005 (2001) 
Cai, R.-G., Nie, Z.-Y., Sun, Y.-W.: Phys. Rev. D 78, 126007 (2008)

Chemissany, W.A., De Roo, M., Panda, S.: Class. Quantum Gravity 25(22), 225009 (2008)

Cuzinatto, R.R., Melo, C.A.M., Medeiros, L.G., de Pompeia, P.J.: Int. J. Mod. Phys. A 26(21), 3641 (2011)

De Lorenci, V.A., Figueiredo, N., Fliche, H.H., Novello, M.: Astron. Astrophys. 369(2), 690 (2001)

De Lorenci, V.A., Klippert, R., Novello, M., Salim, J.M.: Phys. Rev. D 65(6), 063501 (2002)

De Melo, C.A.M., Medeiros, L.G., Pompeia, P.J.: Mod. Phys. Lett. A 30(6), 1550025 (2015)

De Oliveira, H.P.: Class. Quantum Gravity 11(6), 1469 (1994)

Delphenich, D.H.: arXiv:hep-th/0309108 (2003, preprint)

Delphenich, D.H.: arXiv:hep-th/0610088 (2006, preprint)

Demianski, M.: Found. Phys. 16(2), 187 (1986)

Diaz-Alonso, J., Rubiera-Garcia, D.: J. Phys. Conf. Ser. 314, 012065 (2013a)

Diaz-Alonso, J., Rubiera-Garcia, D.: Gen. Relativ. Gravit. 45(10), 1901 (2013b)

Diaz-Alonso, J., Rubiera-Garcia, D.: Phys. Rev. D 82(8), 085024 (2010a)

Diaz-Alonso, J., Rubiera-Garcia, D.: Phys. Rev. D 81(6), 064021 (2010b)

Dunne, G.V.: From fields to strings: circumnavigating theoretical physics. In: Heisenberg-Euler Effective Lagrangians: Basics and Extensions, vol. 1, p. 445 (2005)

Dyadichev, V.V., Galtsov, D.V., Zorin, A.G., Zotov, M.Y.: Phys. Rev. D 65(8), 084007 (2002)

Fradkin, E.S., Tseytlin, A.A.: Phys. Lett. B 163(1), 123 (1985)

Garcia-Salcedo, R., Breton, N.: Int. J. Mod. Phys. A 15(27), 4341 (2000)

Gunasekaran, S., Kubiznák, D., Mann, R.B.: J. High Energy Phys. 1211(11), 110 (2012)

Heisenberg, W., Euler, H.: Z. Phys. 98(11-12), 714 (1936)

Hendi, S.H., Momennia, M.: Eur. Phys. J. C 75, 54 (2015)
Hendi, S.H., Panahiyan, S.: Phys. Rev. D 90, 124008 (2014)

Hendi, S.H., Vahidinia, M.H.: Phys. Rev. D 88(8), 84045 (2013)

Hendi, S.H., Panahiyan, S., Mahmoudi, E.: Eur. Phys. J. C 74, 3079 (2014)

Leigh, R.G.: Mod. Phys. Lett. A 4(28), 2767 (1989)

Linares, R., Maceda, M., Martínez-Carbajal, D.: (2014, preprint)

Lorenci, V.A.D., Souza, M.A.: Phys. Lett. B 512(3-4), 417 (2001)

Medeiros, L.G.: Int. J. Mod. Phys. D 21(09) (2012)

Metsaev, R.R., Rahmanov, M.A., Tseytlin, A.A.: Phys. Lett. B 193(2), 207 (1987)

Mo, J.X., Liu, W.B.: Eur. Phys. J. C 74, 2836 (2014)

Moniz, P.V.: Phys. Rev. D 66(10), 103501 (2002)

Niau-Akmansoy, P., Medeiros, L.G.: Phys. Lett. B 738, 317 (2014)

Novello, M., Bittencourt, E.: Phys. Rev. D 86(12), 124024 (2012)

Novello, M., Goulart, E.: Eletrodinâmica Não Linear. Causalidade e Efeitos Cosmológicos. Livraria da Física, São Paulo (2010)

Novello, M., Araujo, A.N., Salim, J.M.: Int. J. Mod. Phys. A 24(30), 5639 (2009)

Novello, M., Bergliaffa, S.E.P., Salim, J.: Phys. Rev. D 69(12), 127301 (2004)

Novello, M., De Lorenci, V.A., Salim, J.M., Klippert, R.: Phys. Rev. D 61(4), 045001 (2000)

Novello, M., Perez Bergliaffa, S.E., Salim, J., De Lorenci, V., Klippert, R.: Class. Quantum Gravity 20, $859(2003)$

Rasheed, D.A.: arXiv:hep-th/9702087 (1997, preprint)

Ruffini, R., Wu, Y.-B., Xue, S.-S.: Phys. Rev. D 88(8), 085004 (2013)

Salazar, H., García, A., Plebański, J.: Nuovo Cimento B 84(1), 65 (1984)

Schwinger, J.: Phys. Rev. 82, 664 (1951)

Seiberg, N., Witten, E.: J. High Energy Phys. 1999(09), 032 (1999)

Stehle, P., DeBaryshe, P.G.: Phys. Rev. 152, 1135 (1966)

Tu, L.-C., Luo, J., Gillies, G.T.: Rep. Prog. Phys. 68(1), 77 (2005)

Weinberg, S.: Gravitation and Cosmology: Principle and Applications of General Theory of Relativity. Wiley, New York (1972)

Yajima, H., Tamaki, T.: Phys. Rev. D 63(6), 064007 (2001) 